\title{
Interrupção do Arco Aórtico: Tratamento Cirúrgico no Adulto em um Centro de Referência de Cardiopatia
}

\author{
Aortic Arch Interruption: Adult Surgical Treatment at a \\ Reference Center for Adult Heart Disease
}

\author{
Bruno da Costa Rocha, Breno Soares Wanderley, Anabel Góes Costa \\ Serviço de Cardiopatia Congênita no Adulto do Hospital Santa Izabel; \\ Salvador, Bahia, Brasil
}

Correspondence addresses: Dr. Bruno Rocha brunorochaccv@hotmail.com

Received: November 13, 2018

Revised: December 12, 2018

Accepted: January 22, 2019

Published: March 27, 2019

Data Availability Statement: All relevant data are within the paper and its Supporting Information files.

Competing interests: The authors have declared that no competing interests exist.

\section{Copyright}

(C) 2019 by Santa Casa de Misericórdia da Bahia.

All rights reserved.

ISSN: 2526-5563
A interrupção do arco aórtico (IAA) é uma condição extremamente rara, caracterizada pela interrupção luminal segmentar ou focal. $O$ diagnóstico pormenorizado é realizado por angiotomografia computadorizada (AngioTC) ou ressonância nuclear magnética (RNM) e o tratamento e eminentemente cirúrgico. Entre 2013 e início de 2019, 4 pacientes adultos foram submetidos à cirurgia para tratamento de IAA tipo A no Hospital Santa Izabel (HSI) através da interposição cirúrgica de enxerto por via extra-anatômica, entre a aorta torácica ascendente e descendente, por via mediana. A mediana de permanência dos pacientes na Unidade de Terapia Intensiva (UTI) foi de 3 dias, hospitalar de 7 dias, tempo de acompanhamento médio de 31,2 meses $\pm 25,3$, não havendo nenhuma complicação hemodinâmica, infecciosa ou óbito. Em comparação com outros centros de referência, como o Texas Heart Institute (USA), a técnica de tratamento cirúrgica aplicada no HSI demonstrou-se segura, sem mortalidade e com resultados a médio prazo adequados e compatíveis com a literatura.

Palavras-chave: Aorta Torácica (anormalidades); Interrupção de arco aórtico; Xenoenxerto; HipertensãoArterial Sistêmica.

Aortic arch interruption (IAA) is an extremely rare condition, characterized by segmental or focal luminal disruption. The detailed diagnosis is performed by computerized angiography (AngioTC) or nuclear magnetic resonance (MRI) and the treatment is eminently surgical. Between 2013 and early 2019, 4 adult patients underwent surgery to treat type A IAA at Santa Izabel Hospital (HSI) through surgical insertion of an extra-anatomical graft between the ascending and descending thoracic aorta. The median patient stay in the Intensive Care Unit (ICU) was 3 days, hospital permanence 7 days, mean follow-up time of 31.2 months \pm 25.3 , with no hemodynamic, infectious or death complications. Compared with reference centers, such as the Texas Heart Institute (USA), the surgical treatment technique applied at the HSI has been shown to be safe, without mortality and with adequate mid-term results, which was corroborated with the literature.

Keywords: Thoracic aorta; Aortic arch interruption; Heterografts; Hypertension.

\section{Introdução}

A má formação congênita do arco aórtico ocorre pela persistência ou involução inadequada dos arcos branquiais no período embrionário. ${ }^{1}$ A interrupção do arco 
aórtico (IAA) é caracterizada pela interrupção luminal segmentar ou focal, considerada extremamente rara, com aproximadamente três indivíduos afetados por um milhão de nascidos vivos. $^{2}$

A IAA é classificada conforme a localização do segmento comprometido:

Tipo A: após subclávea esquerda (SCE) ou distal; Tipo B: entre a SCE e a carótida esquerda comum (CC); ou

Tipo C: entre a CC e o tronco braquicefálico (TBC) ou proximal.

A IAA pode estar associada à hipoplasia de arco, categorizada por diminuição no calibre em relação à aorta ascendente em $60 \%$ para o arco proximal; $50 \%$ para arco distal e $40 \%$ para o istmo aórtico.

A interrupção de arco (IAA) do tipo Aé a mais encontrada em adultos, compatível com a vida, caso haja fluxo para a aorta descendente via persistência de canal arterial, o que ocorre no período neonatal predominantemente e tardiamente com o desenvolvimento de colaterais sistêmicas entre o segmento proximal e distal à interrupção.

A IAA tipo A está associada a outras cardiopatias congênitas como persistência de canal arterial, comunicação interventricular e valva aórtica bivalvular.

O desenvolvimento de sintomas pode ocorrer e não são patognomônicos, ao exemplo de claudicação intermitente de membros inferiores, isquemia crônica mesentérica, cefaleia e edispneia progressiva. A hipertensão arterial proeminente em membro superior e vasos cerebrais pode evoluir com complicações como hemorragia por rotura de aneurisma cerebral, assim como hipertrofia ventricular esquerda progressiva e morte súbita. ${ }^{3}$

O diagnóstico pormenorizado é realizado por angiotomografia computadorizada (AngioTC) ou ressonância nuclear magnética (RNM), entretanto é geralmente precedido de ecocardiograma bidimensional e avaliação clínica rigorosa que inclui medida de pressão arterial nos quatro membros. A avaliação dos vasos cerebrais por estes métodos pode revelar aneurisma cerebral ou má formação arteriovenosa. O cateterismo cardíaco pode ser solicitado para complementar a avaliação funcional ou cineangiocoronariografia.
O tratamento da IAA tipo A é eminentemente cirúrgico. A técnica com interposição de enxerto sintético tubular entre o segmento proximal à interrupção e distal é o método de escolha no adulto. A interposição de enxerto por via extra-anatômica, entre a aorta torácica ascendente e descendente, por via mediana, é amplamente realizada. ${ }^{4} \mathrm{O}$ Hospital Santa Izabel (HSI) (Salvador, BA, Brasil), por ser referência no tratamento de cardiopatia congênita no adulto, vem desenvolvendo experiência cirúrgica nesta rara condição.

\section{Material e Métodos}

No período de novembro de 2013 a janeiro de 2019, 4 pacientes adultos foram submetidos no Hospital Santa Izabel à cirurgia para tratamento de IAA tipo A,utilizando a técnica cirúrgica descrita acima. A técnica consiste em interposição de enxerto tubular sintético entre a aorta ascendente e a descendente por via mediana, passando o enxerto pela face diafragmática e anterior à veia cava inferior com auxílio de circulação extracorpórea (CEC).

O preparo na sala de cirurgia incluiu a monitorização completa padrão e suplementada com temperatura esofágica e retal, pressão invasiva em artéria femoral comum e radial direita contínuas, sendo os pacientes submetidos a anestesia geral balanceada.

A cirurgia consistiu em entrada em CEC e dissecção da aorta descendente por abertura posterior do pericárdio com deslocamento do coração para o espaço pleural direito, criteriosa hemostasia com ligadura de múltiplas colaterais no segmento da aorta acima do diafragma por segmento de aproximadamente $10 \mathrm{~cm}$. Realizada hipotermia leve a profunda, clampeamento da aorta ascendente e cardioplegia sanguínea ou Custodiol.

Realizou-se o clampeamento parcial da aorta descendente e anastomose distal do enxerto sob hipotermia protetora contra isquemia da medula espinhal. Após criteriosa hemostasia com suplemento de cola biológica, retorno do coração à posição habitual e medida do enxerto (entre 22 e $25 \mathrm{~cm}$ de extensão em média), realizou-se a confecção da anastomose proximal da aorta ascendente, aeração de cavidades e do enxerto e despinçamento da aorta 
com normalização do fluxo coronariano. Foi utilizado sistematicamente suporte de sistema de lavagem de hemácias (Autolog ou CellSaver) para reposição suplementar, o que permitiu evitar transfusão de concentrado de hemácias em dois pacientes. Ao final, saída de CEC e procedimentos padrão de síntese.

\section{Relato de Caso}

Quatro pacientes foram operados no período de seis anos, sendo 3 do sexo feminino (75\%), com idade média de 25 anos $\pm 4,3$, peso de $70 \mathrm{Kg} \pm 10,1$, IMC de $26 \pm 3,3$, com tempo de CEC de $135 \mathrm{~min} \pm$ 23,8 e pinçamento de aorta de $53,2 \mathrm{~min} \pm 42$.

Paciente 1: JBQ, $57 \mathrm{Kg}, 1 \mathrm{~m} 61 \mathrm{~cm}$, sexo feminino, 23 anos, estudante, oligossintomática, com HAS descoberta na idade adulta, com medidas de PA $(\mathrm{MSD}=160 \times 80 \mathrm{mmHg}$ e $\mathrm{MIE}=120 \times 100 \mathrm{mmHg})$. Anatomia AngioTC: interrupção de aorta distal com grande quantidade de colaterais e aneurisma de $3 \mathrm{~cm}$ em ramo da SCE e istmo aórtico.

Vasos cerebrais normais.

Cirurgia: DacronTerumo 20mm + Secção de aneurisma istmo aórtico. CEC 150min; pinçamento de aorta $=18 \mathrm{~min}$ em hipotermia profunda.

Follow-up: 5 anos e 2 meses assintomática

Uso de beta-bloqueador por 12 meses de PO, diminuído e suspenso após MAPA 24h normal.

Método de reavaliação: RNM enxerto normal e aneurisma resolvido.

Paciente 2: CCJ, 25 anos, $81 \mathrm{Kg}, 1 \mathrm{~m} 64 \mathrm{~cm}$, sexo feminino, 25 anos, comerciária, observou HAS durante gravidez. Dispneia progressiva, CF II NYHA em uso de BRA e ECA. Tem asma brônquica, com medidas de PA $(\mathrm{MSD}=220 \times 80 \mathrm{mmHg}$ e $\mathrm{MIE}=$ 120x100mmHg).

Anatomia AngioTC: interrupção de aorta distal com grande quantidade de colaterais sem aneurismas.

Vasos Cerebrais normais.

Cirurgia: PTFE Goretex 18mm. CEC 160min; pinçamento de aorta $=20 \mathrm{~min}$ em hipotermia leve.

Follow-up: 3 anos e 1 mês: assintomática.
Uso de BRA por HAS leve associado a Síndrome Metabólica, com medidas de PA (MSD= 150x80mmHg e $\mathrm{MIE}=154 \times 80 \mathrm{mmHg}$ ).

Método de reavaliação: RNM normal (Figura 1).

Figura 1. Imagem de RNM do paciente 2 em 6 meses de PO, setas apontando o enxerto de Goretex entre a aorta ascendente e descendente via diafragmática e acima da veia cava inferior.

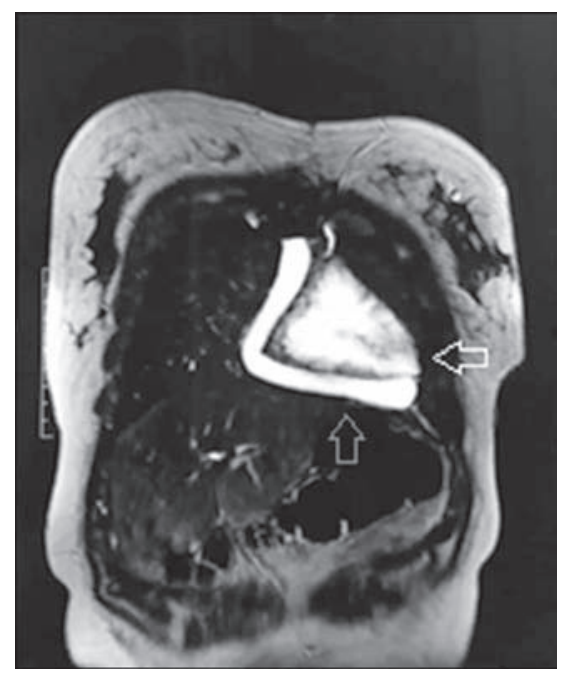

Paciente 3: ECB, 31 anos, $74 \mathrm{Kg}, 1 \mathrm{~m} 70 \mathrm{~cm}$, sexo masculino, carregador, observou HAS durante avaliação de rotina pelo médico do trabalho. Dispneia aos grandes esforços, CF I NYHA, sem medicações, com medidas de PA (MSD = 140x80mmHg e $\mathrm{MIE}=100 \times 90 \mathrm{mmHg}$ ).

Anatomia AngioTC: interrupção de aorta distal com grande quantidade de colaterais sem aneurismas. Vasos Cerebrais normais.

Ecocardiograma: massa de VE 256 (normal até 224g), FE/Ve84\%.

Cirurgia: DacronJotec Flowave 20mm. CEC $120 \mathrm{~min}$; pinçamento de aorta $=105 \mathrm{~min} \mathrm{em}$ hipotermia leve.

Follow-up: 2 anos e 6 meses: assintomático, retornou às atividades laborais intensas como carregador.

Uso de beta-bloqueador por estenose valvar aórtica leve por valva aórtica bivalvular, com medidas de $\mathrm{PA}(\mathrm{MSD}=120 \times 80 \mathrm{mmHg}$ e $\mathrm{MIE}=130 \times 90 \mathrm{mmHg})$. Método de reavaliação: RNM normal. 
Paciente 4: JBA, $68 \mathrm{Kg}, 1 \mathrm{~m} 60 \mathrm{~cm}$, sexo feminino, 21 anos, queixa de cefaleia desde os 5 anos de idade e dispneia progressiva após idade adulta, CF II NYHA em uso de BRA e beta-bloqueador, com medidas de PA $(\mathrm{MSD}=175 \times 115 \mathrm{mmHg}$ e $\mathrm{MIE}=126 \times 90 \mathrm{mmHg}$ ).

Anatomia AngioTC: interrupção de aorta distal com grande quantidade de colaterais sem aneurismas (Figura 2).

Vasos Cerebrais com cerebral média esquerda com discreta dilatação de $2 \mathrm{~mm}$ não aneurismática.

Cirurgia: DacronJotec Flowave 18mm. CEC 110min; pinçamento de aorta $=70 \mathrm{~min}$ em hipotermia leve.

Follow-up: 1 mês (hipotensão ortostática com tontura).

Suspenso no $10 \mathrm{PO}$, beta bloqueador devido a hipotensão, com medidas de $\mathrm{PA}(\mathrm{MSD}=$ 120x80mmHg e $\mathrm{MIE}=115 \times 80 \mathrm{mmHg}$ ).

Método de reavaliação: ecocardiograma com fluxo pulsátil em aorta abdominal.

\section{Resultados e Discussão}

A mediana de permanência dos pacientes na Unidade de Terapia Intensiva (UTI) foi de 3 dias, hospitalar de 7 dias, tempo de acompanhamento médio de 31,2 meses $\pm 25,3$.

Não houve nenhuma complicação hemodinâmica, infecciosa ou óbito.

Figura 2. AngioTC do paciente 4 em reconstrução $3 \mathrm{D}$, demonstrando interrupção de aorta com hipoplasia de arco aórtico.

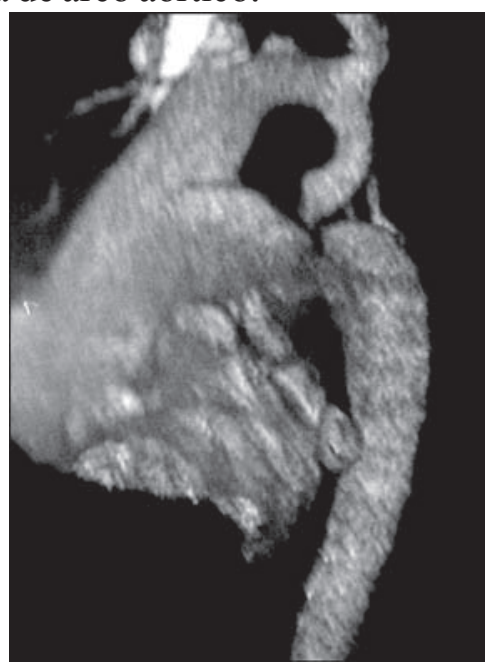

O tratamento cirúrgico no adulto com IAA tipo A é realizado evitando-se as grandes colaterais por acesso mediano, demonstrando-se efetivo para reestabelecer o fluxo pulsátil distal. Na experiência de Krishna e colaboradores ${ }^{4}$, no Texas Heart Institute, com sete pacientes operados, cinco foram abordados por via mediana, sendo descritas complicações naqueles que foram submetidos a via lateral, em especial pela hemorragia e pelo próprio diâmetro inferior dos enxertos 12 e 16mm, em comparação com nossa casuística cujos diâmetros foram de 18 e $20 \mathrm{~mm}^{3}$. A via mediana propicia a circulação extracorpórea plena e hipotermia mais acentuada o que evita a possibilidade de grave complicação como isquemia medular durante clampeamento da aorta descendente podendo levar a paraplegia.

Na casuística do InCor-HCFMUSP de 2001, com 15 pacientes operados, inclusive de coarctação de aorta na idade adulta, $13(86,7 \%)$ foram por via mediana. Nesta série histórica não houve mortalidade assim como a apresentada nesta casuística.

No nosso acompanhamento, três pacientes relataram tontura nos primeiros meses do pósoperatório, provavelmente pelo desvio precoce de sangue para o enxerto que está prévio às artérias do tronco supra-aórtico. Houve ainda relato de estranhamento pela percepção pulsátil em abdome e membros inferiores que coincide em medida não invasiva de PA de pressão de MMII acima da medida de MMSS.

O tipo de enxerto utilizado foi Dacron em 3 pacientes e PTFE em outro, sabendo-se que o custo do PTFE no Brasil é mais alto e por vezes de difícil acesso, em casuística com 4 pacientes operados. Lisboa e colaboradores ${ }^{5}$, no acompanhamento de dois pacientes de 13 e 22 anos, observou estenose não relacionada às anastomoses, cujo material foi Dacron. Segundo Bustos e colaboradores ${ }^{7}$, os enxertos mais recentes de Dacron receberam modificações que conferem resultado anisotrópico analisado em laboratório com capacidade reconhecida. $^{6}$

Apesar de a casuística apresentada ser pequena, outros centros com alto volume de cirurgia para 
tratamento de cardiopatia no adulto, como o Texas Heart Institute, apresentou série com 7 pacientes no período de 5 anos, o InCor-SP, no período de 21 anos, apresentou casuística de 15 pacientes, porém a maioria com o espectro de coarctação de aorta.

Em conclusão, trata-se de um estudo inicial com as evidentes limitações pelo $\mathrm{N}$ apresentado, cuja técnica de tratamento cirúrgica aplicada demonstrou-se segura, sem mortalidade e com resultados a médio prazo adequados e compatíveis com a literatura.

\section{Referências}

1. Priya S, et al. Congenital anomalies of the aortic arch. Cardiovasc Diagn Ther. 2018 Apr; 8(Suppl 1): S26S44.
2. Messner G, et al. Interrupted Aortic Arch in an Adult. Single-Stage Extra-Anatomic Repair. Tex Heart Inst J. 2002;29(2):118-21

3. Vaideeswar P, et al. Discontinuity of the arch beyond the origin of the left subclavian artery in an adult: Interruption or coarctation? Ann Pediatr Cardiol. 2018 Jan-Apr;11(1):92-6.

4. Krishna CS, et al. Interruption of Aortic Arch in Adults: Surgical Experience with Extra-Anatomic Bypass. Tex Heart Inst J. 2005; 32(2):147-50.

5. Lisboa LAF, et al. Tratamento cirúrgico da coarctaçãodo arco aórtico em adulto: avaliaçãoclínica e angiográfica tardia datécnica extra-anatômica. Rev Bras Cir Cardiovasc. 2001;16(3):187-9.

6. Aurigemma D, et al. Non-anastomotic failure of woven Dacron tube grafts in the thoracic aorta in young adults.J Card Surg. 2018 Oct;33(10):65357.

7. Bustos CA, et al. Mechanical characterisation of Dacron graft: Experiments and numerical simulation.J Biomech. 2016 Jan 4;49(1):13-18. 